\title{
Characteristics of Tumors in Lymph Vessels Play an Important Role in the Tumor Progression of Invasive Ductal Carcinoma of the Breast: A Prospective Study
}

\author{
Takahiro Hasebe, M.D., Satoshi Sasaki, M.D., Shigeru Imoto, M.D., Atsushi Ochiai, M.D. \\ Pathology Division (TH, AO) and Epidemiology and Biostatistics Division (SS), National Cancer Center \\ Research Institute East; and Department of Surgery (SI), National Cancer Center Hospital East, Kashiwa, \\ Chiba, Japan
}

It is unknown whether the characteristics of tumor cells in lymph vessels play an important role in the tumor progression of invasive ductal carcinoma (IDC) of the breast. The purpose of this study was to investigate the significance of the characteristics of tumor cells in the lymph vessels in relation to the tumor progression in 393 IDC patients in comparison with well-known histological parameters. The dimensions of lymph vessel tumor emboli were measured, and their structural features, nuclear atypia, and numbers of mitotic and apoptotic figures were also assessed. Multiple regression analysis showed the dimension, the distance, the number of mitotic figures, the number of apoptotic figures, and papillary features of lymph vessel tumor emboli to be significantly associated with the increased number of cells invading the lymph vessels $(P<$ .05). The Cox proportional hazard multivariate analyses showed that more than six apoptotic figures in lymph vessel tumor emboli significantly increased the hazard rates (HRs) of tumor recurrence and death in IDCs without nodal metastasis and that more than four mitotic figures in lymph vessel tumor emboli significantly increased the HRs of tumor recurrence and death in IDCs with nodal metastasis $(P<.05)$. The present study showed that the histological characteristics of tumor cells in lymph

\footnotetext{
Copyright $(2001$ by The United States and Canadian Academy of Pathology, Inc.

VOL. 15, NO. 9, P. 904, 2001 Printed in the U.S.A.

Date of acceptance: May 2, 2002.

This work was supported in part by two Grants-in-Aid from the Ministry of Health and Welfare in Japan, a Grant-in-Aid for Cancer Research and a Grant-in-Aid for the Second Term Comprehensive 10-Year Strategy for Cancer Control.

A portion of this work was presented at the 60th annual meeting of the Japanese Cancer Association on September 27, 2001 in Yokohama, Japan. Address reprint requests to: Atsushi Ochiai, M.D., Pathology Division, National Cancer Center Research Institute East, Kashiwanoha 6-5-1, Kashiwa, Chiba, Japan 277-8577; e-mail: aochiai@east.ncc.go.jp; fax: 81-471-34-6865.

DOI: $10.1097 / 01 . M P .0000024289 .59262 . C E$
}

vessels play a very important role in the tumor progression of IDCs.

KEY WORDS: Breast cancer, Invasive ductal carcinoma, Lymph vessel, Mitosis, Prognosis.

Mod Pathol 2001;15(9):904-913

Invasive ductal carcinomas (IDCs) of the breast are composed of invasive tumor cells, noninvasive tumor cells, and tumor stroma. Some invasive tumor cells are capable of invading lymph vessels, and they are most likely responsible for the lymph node metastasis that is the most important prognostic parameter for IDC patients. However, some IDCs are unassociated with nodal metastasis despite the presence of several invasive lymphatic foci. This strongly suggests that the characteristics or numbers of tumor cells in lymph vessels play an important role in establishing lymph node metastasis by IDCs.

Lymphatic invasion in IDC patients has been reported to have prognostic significance (1-3). However, almost all of the studies have examined only the predictive power of the presence of lymphatic invasion, and no studies have ever investigated the significance of the characteristics or numbers of the tumor cells in lymph vessels as a means of predicting the outcome of IDC patients.

The purpose of this study was to investigate the significance of the characteristics or numbers of tumor cells in lymph vessels in regard to the increase of number of lymph vessels invaded by tumor cells, the presence of lymph node metastasis, the results of tumor recurrence, and tumor death in IDC patients. The results demonstrated that the number of apoptotic figures and the number of mitotic figures in tumor cells in lymph vessels and several other parameters of lymph vessel tumor emboli are independent parameters that predict increased lymphatic invasion, lymph node metastasis, tumor recurrence, and tumor death of IDC 
patients with tumor cell invasion of the lymph vessels.

\section{MATERIALS AND METHODS}

\section{Cases}

Three hundred ninety-three consecutive cases of IDC of the breast surgically treated between July 1992 and November 1998 at the National Cancer Center Hospital East served as the subject of this study. All IDCs were diagnosed by aspiration cytology before surgery. Clinical information was obtained from the patients' medical records after complete histological examination of all IDCs. All patients were Japanese women, and they ranged in age from 28 to 78 years (mean, 51 y). All had a solitary lesion. Two hundred ten patients were premenopausal, and 183 were postmenopausal. Partial mastectomy had been performed in 55, modified radical mastectomy in 314, and standard radical mastectomy in 24. Axillary lymph node dissection consisting of Levels I, II, and \pm III was carried out in all patients. None of the patients had received radiotherapy or chemotherapy before surgery, but 290 patients had received adjuvant therapy. Of the 188 IDC patients without nodal metastasis, 88 had not received adjuvant therapy; 24 had received tamoxifen; 45 had received cyclophosphamide, methotrexate, and 5-FU; Adriamycin and cyclophosphamide; or epirubicin and cyclophosphamide; and 31 had received chemotherapy plus tamoxifen. Of the 205 IDC patients with nodal metastases, 15 had/not received adjuvant therapy, 34 had received tamoxifen, 51 had received chemotherapy, and 105 had received chemotherapy plus tamoxifen. There were no cases of inflammatory breast cancer in this series. All tumors were classified according to the TNM pathologic classification (4). Estrogen receptors (ERs) and progesterone receptors (PRs) in the cytosol fractions were determined by enzyme immunoassay (Otsuka Assay Laboratory, Tokushima, Japan). The upper cutoff values of the ER and PR assays were $13 \mathrm{fmol} / \mathrm{mg}$ protein and $10 \mathrm{fmol} / \mathrm{mg}$ protein, respectively.

For pathological examination, the surgically resected specimens were fixed in $10 \%$ formalin overnight at $4^{\circ} \mathrm{C}$, and the entire tumor was cut into slices at intervals of 0.5 to $0.7 \mathrm{~cm}$. The size and gross appearance of the tumors were recorded, and the former was confirmed by comparison with tumor size on histological slides. Multiple histological sections were taken from each tumor to measure the maximum tumor diameter and area. The mean number and standard deviation of histological sections in each case was $12 \pm 5.8$, ranging from 5 to 49 with a median of 13 . The sections were processed routinely and embedded in paraffin.
Histological Examination of Stroma-Invasive Tumor Cells

Serial sections of each tumor were cut from the paraffin blocks. One section was stained with hematoxylin and eosin and examined pathologically to confirm the diagnosis, and elastica staining was also performed to assess blood vessel invasion in all cases.

The following parameters of primary-invasive tumor cells were examined, and the following cutoff values were established based on univariate analyses using the Cox proportional hazard regression model (5): (1) invasive tumor size ( $\leq 20$ versus $>20$ $\mathrm{mm}$ ), (2) structural features (papillary, cribriform, solid versus strand [scirrhous]; 6), (3) nuclear atypia (mild, moderate versus severe), (4) number of mitotic figures ( $\leq 4$ versus $>4$ ), (5) number of apoptotic figures ( $\leq 16$ versus $>16$ ), (6) tumor necrosis (absent versus present), (7) fibrotic focus (FF), dimension (absent or $\leq 8$ versus $>8 \mathrm{~mm} ; 7-9$ ), and (8) maximum distance of adipose tissue invasion from the tumor margin ( $\leq 2$ versus $>2 \mathrm{~mm}$ ). The number of tumor cell mitotic figures at the most advanced edge of the tumor was counted in 10 high-power fields by the routinely used method (10), and the highest count in the 10 fields was used for comparison with the number of mitotic figures in the tumor cells in the vessels. The count field containing the greatest number of tumor cell apoptotic figures was used (11).

\section{Histological Examination of Tumor Cells in Lymph Vessels}

In accordance with the criteria used to identify lymph vessels (12), in this study we defined tumor cell nests in vessels lined by endothelium with no supporting smooth muscle or elastica as "lymph vessel invasion by tumor cells" (Fig. 1) and tumor cell nests in vessels lined by endothelium that possessed supporting smooth muscle or elastica as "blood vessel invasion." Because artifactual spaces often form around nests of tumor cells within an invasive carcinoma as a result of tissue shrinkage during processing, thus making it very difficult to distinguish these artifacts from true intratumoral lymph vessel spaces, we evaluated lymph vessel invasion at or beyond the border of the stromainvasive tumor area.

The following parameters of tumor cells in lymph vessels were examined with their cutoff values established on the basis of the univariate analyses using the Cox proportional hazard regression model (5): (1) number of lymph vessels invaded (absent or $\leq 5$ versus $>5$ ); (2) number of tumor cells in lymph vessel tumor emboli (disease-free survival, $\leq 100$ versus $>100$; overall survival, $\leq 6000$ versus >6000); (3) maximum dimension of tumor 

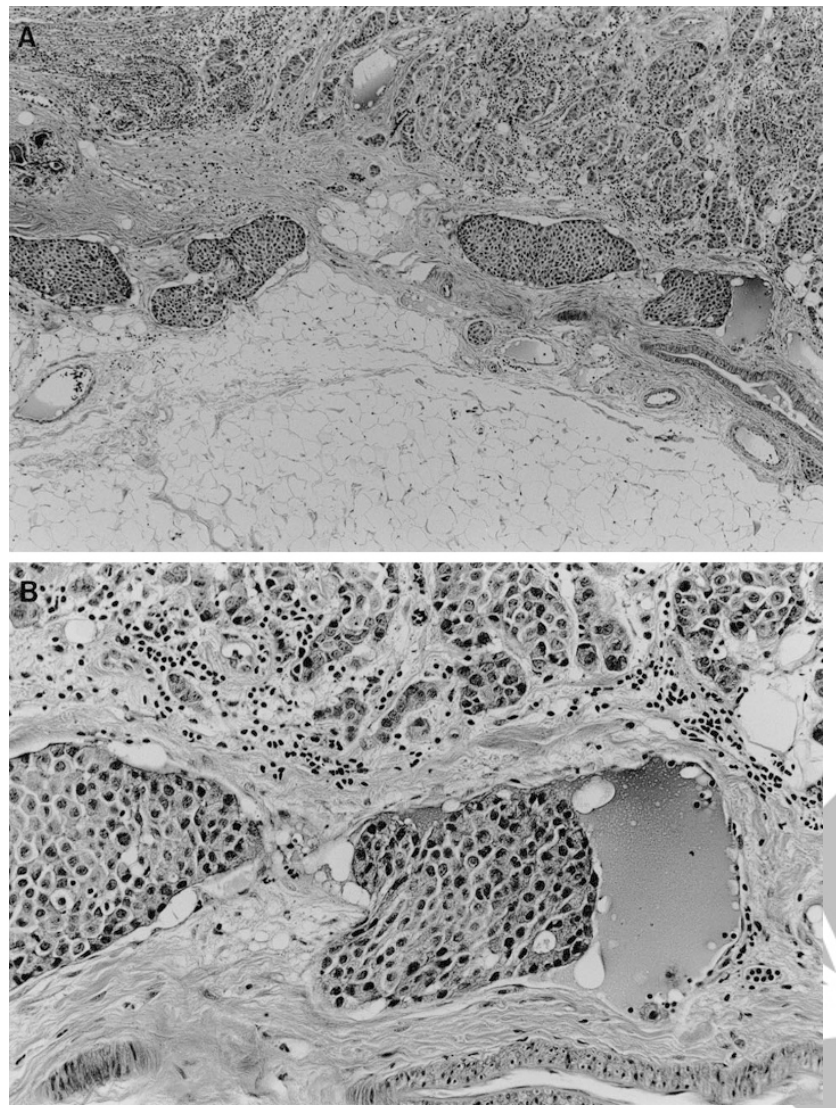

FIGURE 1. A, ductal carcinoma cells in lymph vessels next to an artery and veins. B, the spaces around the clump of tumor cells are lined with endothelium and filled with lymphatic fluid.
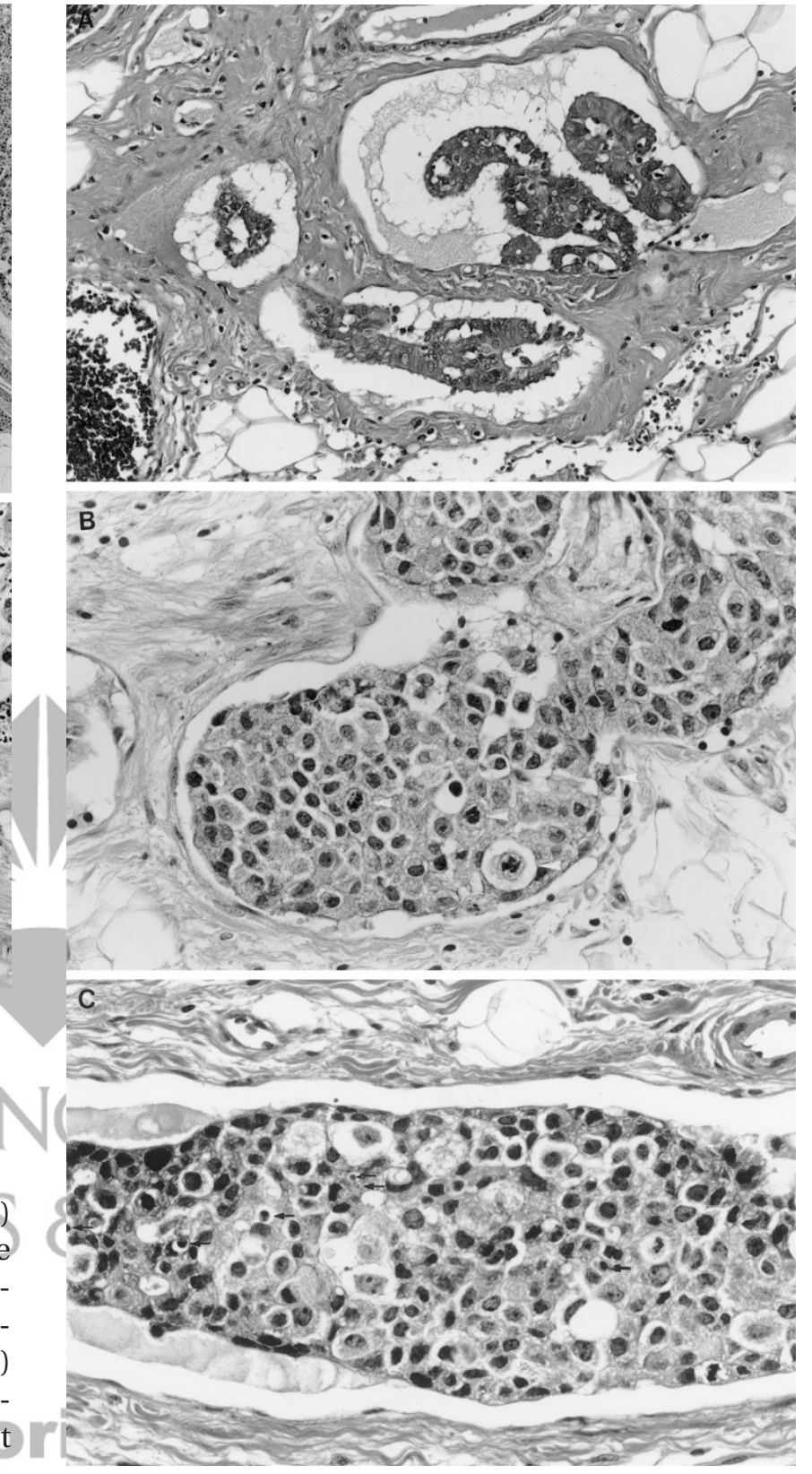

FIGURE 2. A, carcinoma cells within lymph vessels show papillary features. B, carcinoma cells within lymph vessels exhibiting four mitotic figures (arrowheads). C, carcinoma cells within lymph vessels exhibiting six apoptotic figures (arrows).

One author (T.H.) examined all the characteristics of the tumor cells in the lymph vessels, and the other author (A.O.) identified the characteristics of randomly selected IDCs to confirm the characteristics of the tumor cells in the lymph vessels recorded by T.H. Whenever there was a discrepancy, both authors reexamined the slides to reach a consensus.

\section{Outcome}

Patient survival was evaluated by follow-up for a median period of 65 months as of November of 2001. Among the patients as a whole, 95 developed 
tumor recurrence, 64 experienced distant-organ metastasis, and 45 had died of their disease. Metastasis or local recurrence was considered evidence of tumor relapse, and only deaths due to breast cancer were considered for the purposes of this study. Distant-organ metastasis was detected in bone (30 cases), liver (17 cases), lung (17 cases), and brain (4 cases).

\section{Statistical Analysis}

We calculated the maximum, minimum, median, and mean numbers of tumor cells in lymph vessel tumor emboli according to the numbers of lymph vessels invaded within the following number categories: $1,2,3,4$ or 5,6 to 10,11 to 25,26 to 50,51 to 100,101 to 500 , and $>500$.

The multivariate analyses were performed by using the multiple regression model adjusted for parameters of the stroma-invasive tumors and tumor cells in lymph vessels showing a significant correlation with the increase in number of lymph node metastases and with the increase in number of lymph vessels invaded in the univariate analyses. Next, we examined the number of cases according to the following numbers of lymph vessels invaded by tumor cells; 1, 2, 3, 4 and 5, 6 to 10,11 to 25,26 to 50,51 to 100,101 to 500 , and $\geq 501$. We also examined the number of cases in each group belonging to no lymph node metastasis $(n 0), 1$ to 3 lymph node metastases ( $n 1-3), 4$ to 9 lymph node metastases ( $n 4-9)$, and $>9$ lymph node metastases $(n>9)$. Then, we calculated the number of cases belonging to each nodal metastasis class and divided all cases into three groups according to the number of lymph vessel invasion to analyze with the $\chi^{2}$ test whether there were significant differences in the frequencies of $n 1-3, n 4-9$, and $n>9$ among the three groups. In addition, we analyzed the above parameters of stroma-invasive tumors and lymph vessel tumor emboli that were significantly associated with the differences among the three groups by the multivariate analyses using the logistic regression model (13) adjusted for the parameters of the stroma-invasive tumors and lymph vessel tumor emboli. In the multivariate analyses, stroma-invasive tumor size, numbers of mitotic and apoptotic figures in the stroma-invasive tumor, FF dimension, size and distance of lymph vessel tumor emboli, and numbers of mitotic and apoptotic figures in lymph vessel tumor cells were evaluated in real numbers. Mild, moderate, and severe nuclear atypia of stroma-invasive tumor cells and lymph vessel tumor cells were scored as 0,1 , and 2 , respectively, and papillary, cribriform, solid, and strand features were scored as $0,1,2$, and 3 , respectively. Absent/present and negative/positive were scored as 0 and 1, respectively.
Next, we prospectively analyzed the predictive power of the histological characteristics of stromainvasive tumor cells and of the tumor cells in lymph vessels for tumor recurrence, initial distant-organ metastasis, and death in the following groups without knowledge of patient outcomes: (1) a lymph node-negative IDC group and (2) a lymph nodepositive IDC group by multivariate analysis employing the Cox proportional hazard regression model (5) and the step-down method until all remaining factors were significant at a $P$ value of $<$ .05. The multivariate analyses for IDCs without nodal metastasis were adjusted for age ( $\leq 39$ versus $>39 \mathrm{y}$ ), adjuvant therapy (not given versus given), and ER and PR expression (negative versus positive); and the multivariate analyses for IDCs with nodal metastases were adjusted for age, adjuvant therapy, ER and PR status, and nodal status ( $\leq 3$ versus $>3$ ). Curves of disease-free survival, initial distant-organ metastasis-free survival and overall survival were drawn by the Kaplan-Meier method (14).

All analyses were performed with Statistica/Windows software (StatSoft, Tulsa, OK).

\section{RESULTS}

\section{Numbers of Tumor Cells in Lymph Vessel Tumor Emboli}

The numbers of tumor cells in lymph vessel tumor emboli ranged from 9 to 339,625 , and there was à widê range of minimum and maximum numbers of tumor cells in tumor emboli in IDCs with different number of lymph vessels invaded (Table 1). In IDCs with invasion of one to three lymph vessels, the maximum numbers of tumor cells in the lymph vessel tumor emboli was approximately 80 times as large as the minimum number of tumor cells in lymph vessel tumor emboli. Similarly, the maximum numbers of tumor cells in lymph vessel tumor emboli in IDCs with invasion of four or of five or more lymph vessels were about 10 times

TABLE 1. Maximum, Minimum, and Median Numbers of Tumor Cells in Lymph Vessels According to Number of Lymph Vessels Invaded

\begin{tabular}{|c|c|c|c|c|}
\hline \multirow[t]{2}{*}{ No. of Lymph Vessels } & \multirow{2}{*}{$\begin{array}{c}\text { Cases } \\
(N=209)\end{array}$} & \multicolumn{3}{|c|}{$\begin{array}{l}\text { No. of Tumor Cells in Lymph } \\
\text { Vessels }\end{array}$} \\
\hline & & Maximum & Minimum & Median \\
\hline 1 & 42 & 677 & 9 & 49 \\
\hline 2 & 21 & 800 & 14 & 80 \\
\hline 3 & 21 & 321 & 18 & 120 \\
\hline $4-5$ & 16 & 1619 & 111 & 292 \\
\hline $6-10$ & 27 & 1463 & 112 & 352 \\
\hline $11-25$ & 32 & 5563 & 232 & 1191 \\
\hline $26-50$ & 18 & 5851 & 906 & 3030 \\
\hline $51-100$ & 12 & 21,074 & 3091 & 5261 \\
\hline $101-500$ & 13 & 70,030 & 6840 & 19,865 \\
\hline$>500$ & 7 & 339,625 & 27,320 & 81,481 \\
\hline
\end{tabular}


greater than the minimum numbers of tumor cells in lymph vessel tumor emboli in IDCs with the same number of invaded lymph vessels.

\section{Parameters Significantly Associated with Increased Number of Lymph Node Metastases}

Among stroma-invasive tumor parameters, the number of lymph vessels invaded by tumor cells, invasive tumor size, structural features of stromainvasive tumor cells, skin invasion, and the number of apoptotic figures of stroma-invasive tumor cells showed significant associations with increased number of lymph node metastases in the multivariate analysis (Table 2).

\section{Parameters Significantly Associated with} Increased Numbers of Lymph Vessels Invaded by Tumor Cells

Multivariate analysis demonstrated that there were significant associations between size, distance, mitotic figures in lymph vessel tumor emboli, stroma-invasive tumor size, and increased lymph vessels invaded by tumor cells (Table 2). The structural features of lymph vessel tumor emboli and the apoptotic figures in tumor cells in the lymph vessels showed significant inverse correlations with the increased number of lymph vessels invaded by tumor cells. by tumor cells, the percentage of cases with 1 to 9 lymph node metastases from all cases was $>60 \%$ in these groups (Fig. 3A). In IDCs with $\geq 26$ or more lymph vessels invaded by tumor cells, cases with $\geq 10$ nodal metastases reached $60 \%$ in total.

We divided IDCs into the following three groups according to the number of lymph vessels invaded by tumor cells: (1) IDCs with 1 to 3 lymph vessels invaded; (2) IDCs with 4 to 25 lymph vessels invaded; and (3) IDCs with $\geq 26$ lymph vessels invaded (Fig. 3B). Each number of cases belonging to LN0, LN1-3, LN4-9, and LN $>9$ significantly increased according to the increased number of lymph vessels invaded by tumor cells in IDCs $(P<.001)$.

In the multivariate analysis, IDCs with 4 to 25 lymph vessels invaded by tumor cells showed significantly higher relative risks of dimension and distance of lymph vessel tumor emboli, the number of mitotic figures of lymph vessel tumor emboli, and structural and nuclear features of stromainvasive tumors than those with 1 to 3 lymph vessels invaded by tumor cells (Table 3 ). Between IDCs with 4 to 25 and $\geq 26$ lymph vessels invaded by tumor cells, the latter showed significantly higher relative risks of dimension, number of mitotic figures, and distance of lymph vessel tumor emboli, and stroma-invasive tumor size than the former.
Different Status of Nodal Metastases in IDCs According to Number of Invaded Lymph Vessels

In IDCs with one to three lymph vessels invaded by tumor cells, cases without nodal metastasis occupied about half the cases in these groups, whereas in IDCs with 4 to 25 lymph vessels invaded
Mean Mitotic and Apoptotic Figures Values

Primary-invasive tumors showed higher numbers of mitotic and apoptotic figures than tumor cells in lymph vessels in IDCs (Table 4). There was a significant correlation between the number of tumor cell mitotic figures and the number of tumor cell apoptotic figures in the same tumor components.

TABLE 2. Multiple Regression Analyses for Parameters Significantly Associated with Increased Number of Nodal Metastases and with Increased Degree of Lymph Vessel Invasion

\begin{tabular}{|c|c|c|c|}
\hline Parameters & $\mathrm{e}^{\beta}$ & $\begin{array}{l}\text { 95\% Confidence } \\
\text { Interval }\end{array}$ & $P$ Value \\
\hline \multicolumn{4}{|c|}{ Increased no. of nodal metastases (all cases, $n=393$ ) } \\
\hline No. of Ly & 1.4 & $1.3-1.5$ & $<.001$ \\
\hline Invasive tumor size (mm) & 1.3 & $1.2-1.5$ & $<.001$ \\
\hline Structural features of ST & 1.2 & $1.1-1.3$ & $<.001$ \\
\hline Skin invasion & 1.1 & $1.0-1.2$ & .003 \\
\hline Number of AF in ST & 0.9 & $0.8-0.9$ & .019 \\
\hline \multicolumn{4}{|c|}{ Increased degree of lymph vessel invasion (cases with Ly, $n=209$ ) } \\
\hline Dimension of LVTE (mm) & 1.5 & $1.3-1.6$ & $<.001$ \\
\hline Distance of Ly & 1.4 & $1.3-1.6$ & $<.001$ \\
\hline Invasive tumor size (mm) & 1.2 & $1.1-1.3$ & $<.001$ \\
\hline No. of MF in LVTE & 1.2 & $1.1-1.3$ & $<.001$ \\
\hline Structural features of LVTE & 0.8 & $0.8-0.9$ & $<.001$ \\
\hline No. of AF in LVTE & 0.9 & $0.8-0.9$ & .003 \\
\hline
\end{tabular}

No. of mitotic figures and apoptosis of tumor cells of lymph vessel tumor emboli are in one high-power field (400 $\times$ ). The multivariate analyses were performed by the multiple-regression model adjusted for parameters showing a significant correlation with no. of lymph vessels invaded in the univariate analyses. The step-down method was used in the multivariate analysis until all the remaining factors were significant at a $P$ level of $<.05$. Ly, lymph vessel invasion; ST, stroma-invasive tumor cells; AF, apoptotic figures; LVTE, lymph vessel tumor emboli; distance of Ly, greatest distance of lymph vessel tumor emboli from the stroma-invasive tumor margin; MF, mitotic figures. 


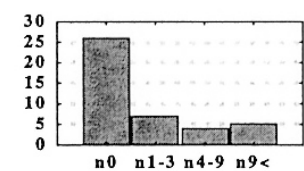

Ly 1

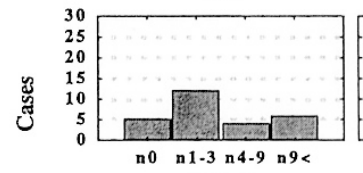

Ly 6-10

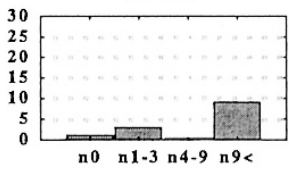

Ly 101-500

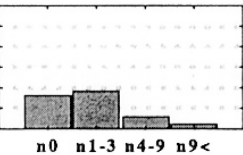

Ly 2

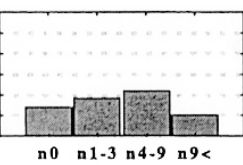

Ly 11-25

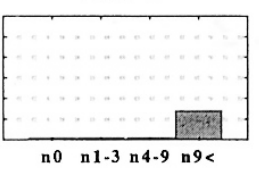

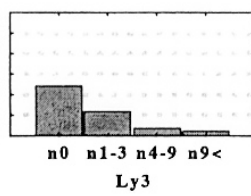

Ly 3

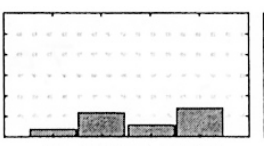

n0 n1-3 n 4-9 n9<

Ly 26.50

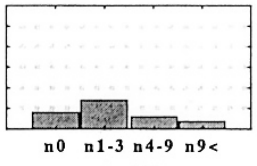

Ly $4 / 5$

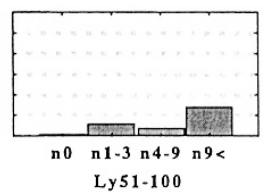

Correlations between Lymph Vessel Invasions and Nodal Metastases

\begin{tabular}{lrrrrr}
\hline & \multicolumn{5}{c}{ No. of patients (\%) } \\
\cline { 2 - 6 } \multicolumn{2}{c}{ Cases } & LN0 & LN 1-3 & LN4-9 & LN9< \\
\hline \multicolumn{2}{l}{ No. of lymph vessel invasion } & & & \\
$1-3$ & 84 & $46(55)$ & $22(26)$ & $9(11)$ & $7(8)$ \\
$4-25$ & 75 & $16(21)$ & $28(37)$ & $18(24)$ & $13(17)$ \\
$>25$ & 50 & $3(6)$ & $12(24)$ & $5(10)$ & $30(60)$ \\
\hline Total & 209 & 65 & 62 & 32 & 50 \\
\end{tabular}

FIGURE 3. A, histograms of the number of nodal metastases according to the number of lymph vessels invaded by tumor cells. In invasive ductal carcinomas (IDCs) with 1 to 3 lymph vessels invaded by tumor cells, cases without nodal metastasis occupied about half of the cases in these groups, whereas in IDCs with 4 to 25 lymph vessels invaded by tumor cells, the percentage of cases with 3 to 9 lymph node metastases in whole cases was $>60 \%$. In IDCs with $\geq 26$ lymph vessels invaded by tumor cells, cases with $\geq 10$ nodal metastases reached $60 \%$ in total. B, the number of cases belonging respectively to LN0, LN1-3, LN4-9, and LN $>9$ was significantly increased according to the increased number of lymph vessels invaded by tumor cells in IDCs $(P<.001)$.

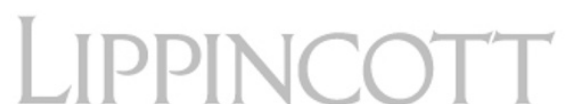

TABLE 3. Multivariate Analyses for Parameters that Were Significantly Different between Invasive Ductal Carcinomas (IDCs) with 1 to $3(n=84)$ and with 4 to 25 Lymph Vessel Invasions $(n=75)$ and between IDCs with 4 to 25 and with $\geq 26$ Lymph Vessel Invasions $(n=50)$

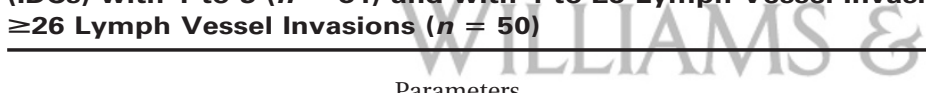

$$
\text { Parameters }
$$

RR Interval

$P$ Value

IDCs with 1 to 3 versus those with 4 to 25 lymph vessel invasions Dimension of LVTE (mm) Structural features of ST

No. of MF in LVTE

Nuclear features of ST

Distance of Ly

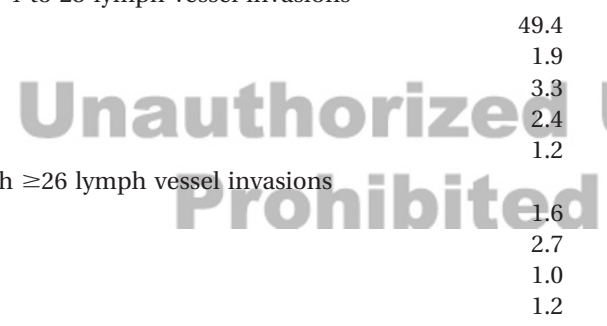

49.4
1.9
3.3
2.4
1.2
1.6
2.7
1.0
1.2

$8.8-277.2$
$1.3-3.0$
$1.2-3.6$
$1.2-4.8$
$1.0-1.5$

$1.2-2.1$
$1.3-6.0$
$1.0-1.1$
$1.0-1.3$

$$
\begin{array}{r}
<.001 \\
.002 \\
.012 \\
.019 \\
.029 \\
\\
.003 \\
.012 \\
.025 \\
.028
\end{array}
$$

No. of MF in LVTE

Dimension of LVTE (mm)

Distance of Ly

$1.0-1.3$

No. of mitotic figures and apoptosis of tumor cells of lymph vessel tumor emboli were in one high-power field (400 $\times$ ). The multivariate analysis was performed by the logistic regression model adjusted for parameters showing a significant correlation with 4 to 25 lymph node metastases or $\geq 26$ lymph node metastases in the univariate analyses. The step-down method was used in the multivariate analysis until all the remaining factors were significant at a $P$ level of $<.05$. RR, relative risk; Ly, lymph vessel invasion; LVTE, lymph vessel tumor emboli; ST, stroma-invasive tumor cells; MFs, mitotic figures. Distance of lymph vessel tumor emboli, greatest distance of lymph vessel tumor emboli from the stroma-invasive tumor margin.

\section{Multivariate Analyses for Tumor Recurrence, Initial Distant-Organ Metastasis, and Tumor Death}

In IDCs without nodal metastasis, more than six apoptotic figures in tumor cells in lymph vessels significantly increased the HRs of tumor recurrence, initial distant-organ metastasis, and tumor death in the multivariate analyses (Table 5; Fig. 4,
A-C). IDCs with FF of $>8 \mathrm{~mm}$ dimension had significantly increased HRs of tumor recurrence and initial distant-organ metastasis in the multivariate analyses. More than four mitotic figures in stromainvasive tumors significantly increased the HR of tumor recurrence, and ER-negative status significantly increased the HRs of tumor death in the multivariate analyses. 
TABLE 4. Mean Values and Standard Deviations of Numbers of Mitotic and Apoptotic Figures in Each Tumor Component of Invasive Ductal Carcinomas with Lymph Vessels Invaded ( $n=209)$

\begin{tabular}{lccc}
\hline \multicolumn{1}{c}{ Component } & $\begin{array}{c}\text { No. of MF } \\
(\text { Mean } \pm \mathrm{SD})\end{array}$ & $\begin{array}{c}\text { No. of AF } \\
(\text { Mean } \pm \mathrm{SD})\end{array}$ & $R, P$ Values \\
\hline Primary invasive tumors & $5.1 \pm 4.4$ & $14.4 \pm 22.9$ & $0.54,<.001$ \\
Lymph vessel tumor emboli & $1.7 \pm 2.5$ & $9.2 \pm 18.4$ & $0.65,<.001$ \\
\hline \multicolumn{1}{c}{ The Spearman's correlations were between mitotic and apoptotic fig- } \\
ures of tumor cells in the same tumor component. MF, mitotic figures; AF, \\
apoptotic figures.
\end{tabular}

In IDCs with nodal metastasis, more than four mitotic figures in tumor cells in lymph vessels, more than four mitotic figures in stroma-invasive tumors, and PR-negative status significantly increased the HRs of tumor recurrence and tumor death in the multivariate analyses (Table 5; Fig. 4, D and F). More than five lymph vessels invaded and $>20$ tumors of invasive size significantly increased the HRs of tumor recurrence in the multivariate

TABLE 5. Multivariate Analyses for Tumor Recurrence, IDOM, and Tumor Death in IDCs Without and With Lymph Node Metastasis

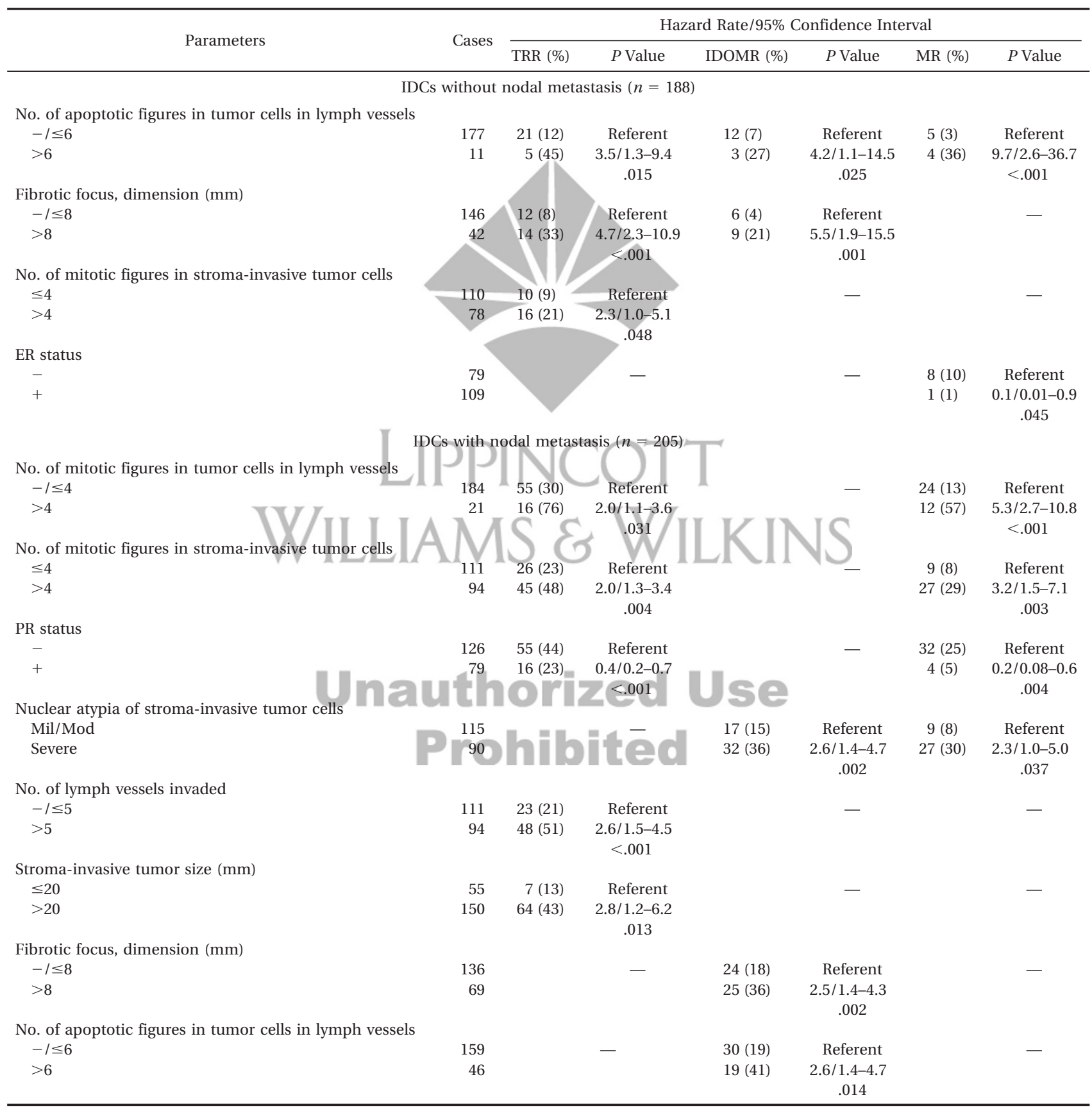

The multivariate analyses for TRR, IDOMR, and MR were performed employing the Cox proportional hazard regression model adjusted for parameters significantly associated with TRR, IDOMR, and MR in the univariate analyses. The step-down method was used in the multivariate analysis until all the remaining parameters were significant at a $P$ level of $<.05$. IDOM, initial distant organ metastasis; IDC, invasive ductal carcinoma; TRR, tumor recurrence rate; IDOMR, IDOM rate; MR, mortality rate; ER, estrogen receptor; PR, progesterone receptor; +, positive; -, absent or negative; Mil, mild; mod, moderate. 
analyses. FF of $>8 \mathrm{~mm}$ dimension and more than six apoptotic figures in tumor cells in lymph vessels (Fig. 4E) significantly increased the HRs of initial distant-organ metastasis in the multivariate analyses.

\section{DISCUSSION}

This study clearly demonstrated that the number of tumor cells in lymph vessel tumor emboli is widely distributed in IDC groups that had almost the same number of invaded lymph vessels. This strongly suggests that the differences between the characteristics of the tumor cells in the lymph vessel tumor emboli caused a wide distribution of the number of tumor cells in the lymph vessels in IDCs with almost the same degree of lymph vessel invasion.

An increased number of lymph vessels invaded by tumor cells showed a significant association with the increased number of nodal metastasis with the highest RR in the multivariate analysis, and almost all parameters that were significantly associated with the increased number of lymph vessels invaded were the characteristics of the tumor cells in the lymph vessels. Thus, the increased numbers of nodal metastases and lymphatic vessel invasion most likely depend on the characteristics of the tumor cells in the lymph vessels, not on those of the stroma-invasive tumor cells. The dimensions of the lymph vessel tumor emboli probably suggest that the number and quantity of tumor cells
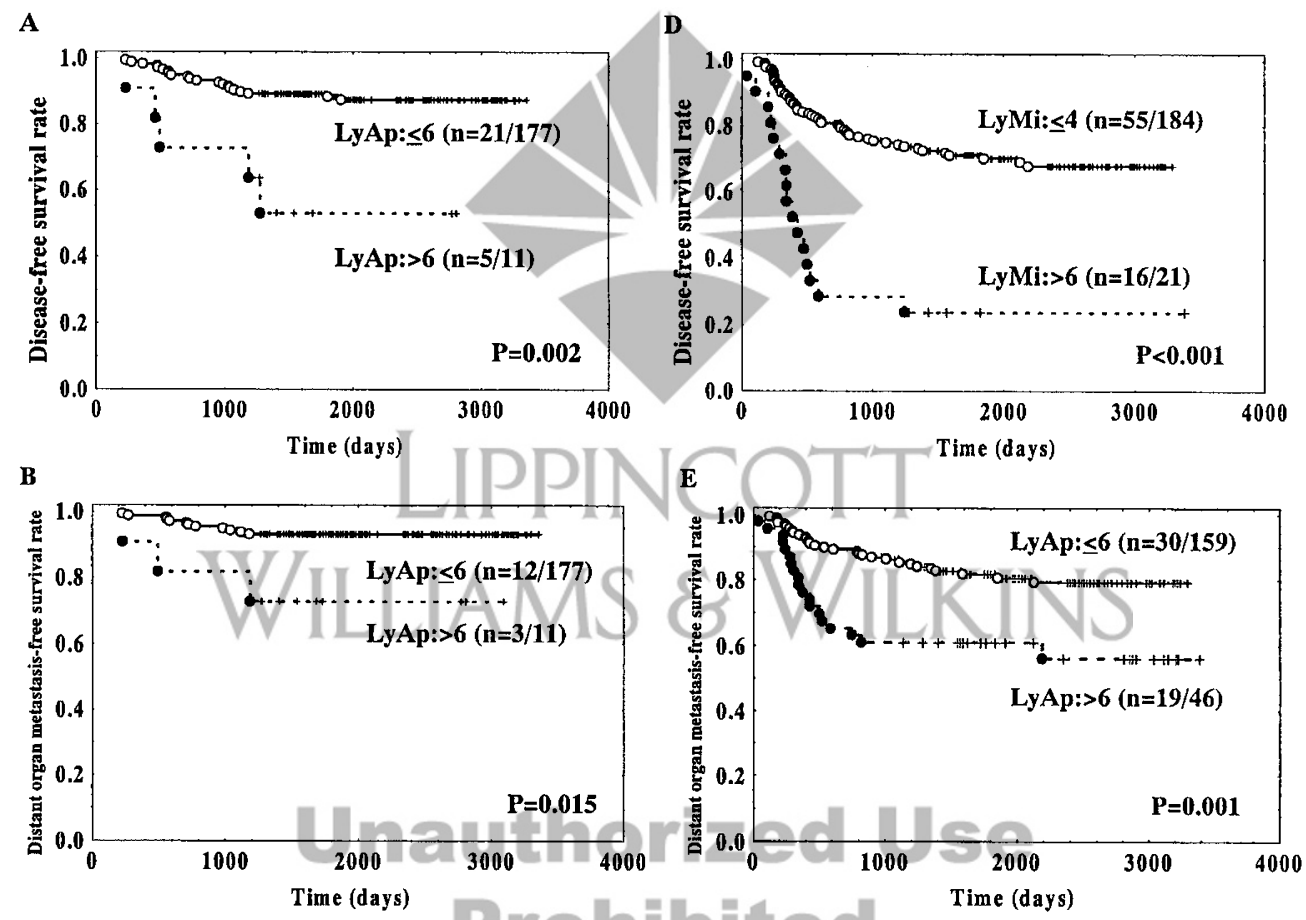

C
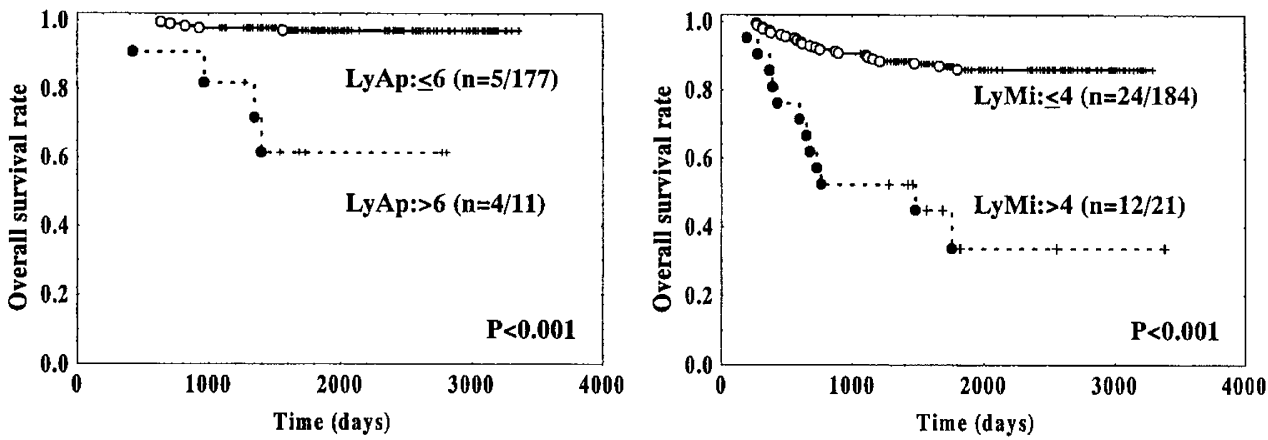

FIGURE 4. A-C, in IDCs without nodal metastasis, IDCs with more than six apoptotic figures in lymph vessel tumor emboli showed significantly shorter disease-free survival periods, initial distant-organ metastasis-free survival periods, and overall survival periods than those with no more than six apoptotic figures in lymph vessel tumor emboli $(P=.002, P=.015$ and $P<.002)$. D \& F, in IDCs with nodal metastasis, IDCs with more than four mitotic figures in lymph vessel tumor emboli showed significantly shorter disease-free survival and overall survival periods than did those with no more than four mitotic figures in lymph vessel tumor emboli $(P<.001$ and $P<.001)$. E, in IDCs with nodal metastasis, IDCs with more than six apoptotic figures in lymph vessel tumor emboli showed a significantly shorter initial distant-organ metastasis-free survival period than those with no more than six apoptotic figures in lymph vessel tumor emboli $(P=.001)$. Ly, lymph vessel; Ap, apoptosis; Mi, mitosis. 
in the lymph vessels play an important role in the increased number of lymphatic vessels invaded. The maximum distance of the lymph vessel tumor emboli from the stroma-invasive tumor margin probably suggests the ability of these cells to spread aggressively via the lymph vessels. The number of mitotic figures in the tumor cells in the lymph vessels most likely shows that tumor cells with a high proliferative activity can increase the number of lymphatic vessels invaded. In addition, the number of apoptotic figures displayed a significant inverse effect on the increase in the number of lymphatic vessels invaded, which strongly suggests that those tumor cells in lymph vessels that are immortal and do not undergo apoptosis survive and form lymph vessel tumor emboli. Thus, the spreading, proliferative, and survival capacities of individual tumor cells as well as the number or quantity of the tumor cells in the lymph vessels most likely play an important role in the increased number of lymphatic vessels invaded in IDCs. Among these characteristics of lymph vessel tumor emboli, the number of mitotic figures, dimension, and distance of lymph vessel tumor emboli were also parameters that were significantly different according to the increased numbers of lymph vessel invasion, e.g., IDCs with 1 to 3 and those with 4 to 25 lymph vessel invasions, and IDCs with 4 to 25 and those with $>25$ lymph vessel invasions. In addition, the number of nodal metastasis significantly increased according to increased number of lymph vessels invaded in the current study. Thus, we conclude that the characteristics of lymph vessel tumor emboli are the most important factors that define the status of nodal metastases in IDCs.

Apoptotic and mitotic figures of tumor cells in lymph vessels had better predictive prognostic power than did the numbers of lymph vessels showing invasion or size of lymph vessel tumor emboli. This strongly suggests that metastatic IDC nests in other organs seeded via lymph vessels cannot be established solely on the basis of large numbers of lymph vessels being invaded or large quantities of tumor cells being present in the lymph vessels. The numbers of apoptotic and mitotic figures in tumor cells in lymph vessels probably reflect the characteristics of the individual tumor cells. The two counts in tumor cells in lymph vessels were significantly correlated with each other, and in this study highly apoptotic or mitotic tumor cells in lymph vessels had greater prognostic predictive power than did primary-invasive tumors. Based on these findings, tumor cells with a high turnover rate in lymph vessels probably have a greater ability to form metastatic foci in lymph nodes or distant organs than do primary-invasive tumor cells with a high turnover rate, and they are therefore probably responsible for the lethal effect in IDC patients. From these, the following cutoff values will probably be very useful for predicting outcomes of IDC patients with lymph vessel invasion in routine examination: (1) number of apoptotic figures, 6 and (2) number of mitotic figures, 4.

The number of tumor cells in lymph vessels, however, was not a significant parameter for predicting the outcome of the IDC patients in this study. The values represented the actual total number of tumor cells in lymph vessels at the time of the operation, but not the true total number of tumor cells that spread via the lymph vessel system from the time of the initial occurrence of lymph vessel invasion to the time of operation. For this reason, it seems that the number of tumor cells in lymph vessels did not appear to be a significant prognostic parameter for IDC patients.

The results of this study strongly suggest that the lymph vessel system is a very important tumor metastatic pathway, as well as is the blood vessel pathway and tumor angiogenesis $(15,16)$. It is, therefore, necessary to identify the functions of tumor cells in lymph vessels to prevent their progression via the lymph vessel system as a means of improving the prognosis of patients with IDC of the breast.

\section{REFERENCES}

1. Lauria R, Perrone F, Carlomagno C, De Laurentiis M, Morabito A, Gallo C, et al. The prognostic value of lymphatic and

blood vessel invasion in operable breast cancer. Cancer 1995;76:1772-8.

2. Nime FA, Rosen PP, Tzvi Thaler H, Ashikari R, Urban JA. Prognostic significance of tumor emboli intramammary lymphatics in patients with mammary carcinoma. Am J Surg Pathol 1977;1:25-30.

3. Rosen PP, Groshen S, Saigo PE, Kinne DW, Hellman S. Pathological prognostic factors in stage I (T1NOM0) and stage II (T1N1M0) breast carcinoma: a study of 644 patients with median follow-up of 18 years. J Clin Oncol 1989;7:1239-51.

4. Sobin LH, Witteklnd C. TNM classification of malignant tumors, 5th ed. New York: Wiley-Liss; 1997. p. 123-30.

5. Cox DR. Regression models and life-tables. J R Stat Soc 1972;34:187-220.

6. Sakamoto G. Infiltrating carcinoma: major histological types. In: Page DL, Anderson TJ, editors. Diagnostic histopathology of the breast. Edinburgh: Churchill Livingstone; 1987. p. 193-7.

7. Hasebe T, Tsuda H, Hirohashi S, Shimosato Y, Tsubono Y, Yamamoto $\mathrm{H}$, et al. Fibrotic focus in infiltrating ductal carcinoma of the breast: a significant histopathological prognostic parameter for predicting the long-term survival of the patients. Breast Cancer Res Treat 1998;49:195-208.

8. Hasebe T, Mukai K, Tsuda H, Ochiai A. New prognostic histological parameter of invasive ductal carcinoma of the breast: clinicopathological significance of fibrotic focus. Pathol Int 2000;50:263-72.

9. Hasebe T, Sasaki S, Imoto S, Ochiai A. Highly proliferative fibroblasts forming fibrotic focus govern metastasis of invasive ductal carcinoma of the breast. Mod Pathol 2001;14:325-37.

10. Elston CW, Ellis IO. Pathological prognostic factors in breast cancer. I. The value of histological grade in breast cancer: experience from a large study with long-term follow-up. Histopathology 1991;19:403-10. 
11. Jong JS de, van Diest PJ, Baak JPA. Number of apoptotic cells as a prognostic marker in invasive breast cancer. Br J Cancer 2000;82:368-73.

12. Rosen PP. Breast pathology. Philadelphia, PA: LippincottRaven; 1996. p. 283-5.

13. Truett J, Cornfield J, Kannel W. A multivariate analysis of the risk of coronary heart disease in Framingham. J Chronic Dis 1967;20:511-24.
14. Kaplan EL, Meier P. Nonparametric estimation from incomplete observations. J Am Stat Assoc 1958;53:457-81.

15. Weidner N, Semple JP, Welch WR, Folkman J. Tumor angiogenesis and metastasis: correlation in invasive breast carcinoma. N Engl J Med 1991;324:1-8.

16. Jitsuiki Y, Hasebe T, Tsuda H, Imoto S, Tsubono Y, Sasaki S, et al. Optimizing microvessel counts according to tumor zone in invasive ductal carcinoma of the breast. Mod Pathol 1999;12:492-8.

\section{Book Review}

\section{Layfield LJ: Cytopathology of Bone and Soft Tissue Tumors, 266 pp, Hong Kong, Oxford University Press, 2002 (\$149.50).}

Cytology texts of a single organ system are always a bit chancy for publishers due to perceived limited appeal or sales versus the "profit" motive. When the cytology diagnosis of that organ system has less widespread acceptance for definitive diagnosis and deals with rare entities within general pathology practice, then it can be even more difficult to get a publisher to commit to the idea of "profiting medical knowledge." Thus I must give Oxford University Press credit for bringing us this enlightening and sensible approach to cytology of soft tissue and bone lesions.

Dr. Layfield does an excellent job, starting with a well-written preface and continuing throughout the text of placing conditions and cautions upon the diagnosis of malignancies in this difficult arena of cytology. Chapters are broken into subheadings that are similar to the popular soft tissue surgical pathology texts (by order of differentiation). Unfortunately, one minor deficiency is that by following this type of outline it misses discussing some sarcomatous tumors such as GIST and solitary fibrous tumors that may enter differentials of spindle cell neoplasms for clinical management decisions. However, it is otherwise very comprehensive as to bone and soft tissue lesions. There are excellent tables and flow diagrams that are useful while signing out FNAs that accompany the detailed written descriptions. Accompanying color photos give very satisfactory representation with modified Giemsa stain and Pap stain for smears with H\&E for tissue or cell block preparations. There are outlines of the major diagnostic features for each entity for a quick run-through during sign-out. What is also quite helpful is a section titled "problems in diagnosis" for each entity that is discussed with suggestions for ancillary testing if sufficient sampling exists.

I found it to be quite useful when presented with the occasional fine needle aspiration of bone or soft tissue when identifying something other than the more common confirmation of metastasis of a known primary malignancy. In addition, it was also useful due to its histological correlations for the small core biopsies with accompanying touch preps. It gave a very honest, balanced approach that clearly cautions against overconfideñnce based sōlely on cytological features but gets you into the differential "ball park" with its clearly described cytological features. I was able to find discussion of both nonneoplastic and neoplastic rarer entities that I could not find in comprehensive cytology texts. I would definitely recommend Dr. Layfield's text for all cytology teaching departments as well as for those pathologists that have interventional radiology departments and/or oncological orthopedic surgical departments.

\author{
Larry J. Fowler \\ University of Texas Health Science Center at \\ San Antonio \\ San Antonio, Texas
}

